\title{
BANACH SPACES WITH THE EXTENSION PROPERTY
}

\author{
BY \\ J. L. KELLEY(1)
}

It is the object of this note to complete a characterization of those Banach spaces $B$ with the Hahn-Banach extension property: each bounded linear function $F$ on a subspace of any Banach space $C$ with values in $B$ has a linear extension $F^{\prime}$ carrying all of $C$ into $B$ such that $\left\|F^{\prime}\right\|=\|F\|$. It is shown here that:

Theorem. Each such space $B$ is equivalent to the space $C_{X}$ of continuous real-valued functions on an extremally disconnected compact Hausdorff space $X, C_{X}$ having the usual supremum norm.

Recently, in these Transactions, Nachbin $[\mathrm{N}]$ and, independently, Goodner [G] have shown that if $B$ has the extension property and if its unit sphere has an extreme point, then $B$ is equivalent to a function space of this sort; both authors have also proved that such a function space has the extension property. The above theorem simply omits the extreme point hypothesis, and so establishes the equivalence.

My original proof, of which the proof given here is a distillate, depends on an idea of Jerison [J]. Briefly, letting $X$ be the weak* closure of the set of extreme points of the unit sphere of the adjoint $B^{*}, B$ can be shown equivalent to the space of all weak* continuous real functions $f$ on $X$ such that $f(x)=-f(-x)$, and then properties of $X$ are deduced which imply the theorem. The same idea occurs implicitly in the proof below.

Note. Goodner asks [G, p. 107] if every Banach space having the extension property is equivalent to the conjugate of an abstract $(L)$-space. It is known (this is not my contribution) that the Birkhoff-Ulam example ([B, p. 186] or [HT, p. 490]) answers this question in the negative, the pertinent Banach space being the bounded Borel functions on $[0,1]$ modulo those functions vanishing except on a set of the first category, with $\|f\|$ $=\inf \{K:|f(x)| \leqq K$ save on a set of first category $\}$.

1. Preliminary definitions and remarks. A point $x$ is an extreme point of a convex subset $K$ of a real linear space if $x$ is not an interior point of any line segment contained in $K$ (i.e., if $x=t y+(1-t) z, 0<t<1, y \in K$, and $z \in K$, then $x=y=z$ ). A set $L$ is a support of $K$ if $L$ is a convex, nonvoid subset of $K$ such that each line segment contained in $K$ which has an interior point in $L$ is contained in $L$. If $x$ is an extreme point of $L$ and $L$ is a sup-

Presented to the Society, September 6, 1951; received by the editors July 26, 1951.

(1) This work was done under Contract N 7-onr-434, Task Order III, Navy Department, the Office of Naval Research, U.S.A. 
port of $K$, then $x$ is an extreme point of $K$. If $F$ is a linear function carrying a convex set $K$ into a convex set $M$ and $L$ is a support of $M$, then $F^{-1}(L) \cap K$ is either void or a support of $K$.

For each Banach space $B$ the adjoint space is denoted by $B^{*}$ and the weak ${ }^{*}$ topology for $B^{*}$ is the topology of pointwise convergence of functionals. Each convex, norm-bounded, weak* closed subset $K$ (= convex, weak* compact subset) is, according to the classic theorem of Krein and Milman, the smallest convex weak* closed set which contains all extreme points of $K$. (See, for example, $[K]$.) If $F$ is a bounded linear function on $B$ to a Banach space $C$, then $F^{*}$, the adjoint function, carries $C^{*}$ into $B^{*}$ in a weak ${ }^{*}$ continuous fashion, and in particular, the image of the unit sphere of $C^{*}$ is weak* compact.

A compact Hausdorff space is extremally disconnected if the closure of each open set is open. If $X$ is a compact Hausdorff space, then $C_{X}$ is the Banach space of all real-valued continuous functions on $X$, with the usual supremum norm. For each $x \in X$ there is assigned a functional $e_{x}$, by setting $e_{x}(f)=f(x)$ for $f \in C_{X}$. This functional $e_{x}$ is the evaluation at $x$. It is known (see [AK]) that the set of extreme points of the unit sphere of $C_{X}^{*}$ is precisely $E \cup(-E)$, where $E$ is the set of all evaluations. Moreover, if $E$ has the relativized weak* topology, then the function $e$ carrying $x$ into $e_{x}$ maps $X$ homeomorphically onto $E$.

2. Proof of the theorem. Let $B$ be a Banach space with the property: if $H$ is a linear isometry of $B$ into a Banach space $C$, then there is a linear map $G$ of norm one carrying $C$ onto $B$ such that $G H$ is the identity map of $B$ onto itself. Let $X$ be the weak* closure of the set of all extreme points of the unit sphere of $B^{*}$. Then $X$ is weak* compact. In what follows, a subset of $X$ is "open" if it is "open in the relativized weak* topology for $X$," and the closure $U^{c}$ of a subset $U$ of $X$ is the weak ${ }^{*}$ closure of $U$.

Suppose, now, that $U$ and $V$ are open subsets of $X$ such that both $U \cap V$ and $[-(U \cup V)] \cap(U \cup V)$ are void, and $[-(U \cup V)] \cup(U \cup V)$ is dense in $X$. We construct a space $Y$, by setting $Y=\left(\{0\} \times U^{c}\right) \cup\left(\{1\} \times V^{c}\right)$, so that $Y$ consists of disjoint copies of $U^{c}$ and $V^{c}$. The set $Y$ is topologized by agreeing that if $U_{1}$ is open in $U^{c}$ and $V_{1}$ is open in $V^{c}$, then $\{0\} \times U_{1}$ and $\{1\} \times V_{1}$ are each open in $Y$. Let $H$ be the map of $B$ into $C_{Y}$ defined, for $b \in B, u \in U^{c}$, $v \in V^{c}$ by: $H(b)((0, u))=u(b), H(b)((1, v))=v(b)$. The basic result about this construction is:

Lemma. The map $H$ is a linear isometry of $B$ onto $C_{Y}$. Moreover, $U^{c} \cap V^{c}$ and $\left[-\left(U^{c} \cup V^{c}\right)\right] \cap\left(U^{c} \cup V^{c}\right)$ are void, and $H^{*}$ maps the set of evaluations in $C_{Y}^{*}$ weak* homeomorphically onto $U^{c} \cup V^{c}$.

Proof. We first verify that $H$ is a linear isometry. The unit sphere $S$ of $B^{*}$ is weak ${ }^{*}$ compact and, for each $b \in B$, the linear functional $b^{\prime}$, whose value at $z \in B^{*}$ is $z(b)$, is weak* continuous, and maps $S$ onto the closed interval 
$[-\|b\|,\|b\|]$. The set of points at which the functional $b^{\prime}$ assumes the value $\|b\|$ is a support of $S$ and hence contains an extreme point $x$, which is a member of $X$. Either $x$ or $-x$ belongs to $U^{c} \cup V^{c}$, and consequently $\|H(b)\|$ $\geqq|x(b)|=\|b\|$. On the other hand, since $U^{c} \cup V^{c}$ is a subset of the unit sphere of $B^{*},\|H(b)\| \leqq\|b\|$, so that $H$ is an isometry.

Next, a small calculation. Suppose $e_{(0, u)} \in C_{Y}^{*}$ is the evaluation at $(0, u)$, and that $b \in B$. Then $H^{*}\left(e_{(0, u)}\right)(b)$ is, by definition of $H^{*}, e_{(0, u)}(H(b))$, which from the definition of $e_{(0, u)}$ is $H(b)((0, u))$, and using the definition of $H$ this is $u(b)$. Consequently, the valuation at $(0, u)$ maps under $H^{*}$ onto $u$, and similarly the evaluation at $(1, v)$ maps onto $v$.

If $u \in U$ and $u$ is an extreme point of the unit sphere $S$ of $B^{*}$, then $H^{*-1}(u)$ intersects the unit sphere $T$ of $C_{Y}^{*}$ in a set which is a support of $S$. This support, being weak* compact, consists of a single point or else contains at least two extreme points (the Krein-Milman theorem). Each extreme point of the support is also an extreme point of $T$. But the extreme points of $T$ are \pm evaluations, and since $u \notin V^{c}$, the only extreme point which can map onto $u$ under $H^{*}$ is $e_{(0, u)}$, in view of the preceding paragraph. Consequently, $H^{*-1}(u) \cap T$ consists of the single point $e_{(0, u)}$ and similarly, if $v \in V$ and $v$ is an extreme point of $S$, then $H^{*-1}(v) \cap T=\left\{e_{(1, v)}\right\}$.

Now let $G$ be a linear function of norm one carrying $C_{Y}$ onto $B$ so that $G H$ is the identity on $B$. Then $G^{*}$ carries the unit sphere $S$ of $B^{*}$ into the unit sphere $T$ of $C_{Y}^{*}$ and $(G H)^{*}=H^{*} G^{*}$ is the identity on $B^{*}$. If $u \in U$ and $u$ is an extreme point of $S$, then necessarily $G^{*}(u)=e_{(0, u)}$, in view of the preceding paragraph, and if $v \in V$ and $v$ is an extreme point of $S$, then $G^{*}(v)=e_{(1, v)}$. Because such points are dense in $U$ and in $V$ the function $G^{*}$ carries a dense subset of $X$ onto a weak* dense subset of $E \cup(-E)$, where $E$ is the set of evaluations. Because $X$ and $E \cup(-E)$ are weak* compact $G^{*}$ carries $X$ onto $E \cup(-E)$. Now $H^{*} G^{*}$ is the identity on $B^{*}$, and if $u \in U$ and $u$ is an extreme point of $S$, then $G^{*} H^{*}\left(e_{(0, u)}\right)=G^{*}(u)=e_{(0, u)}$, and similarly for $v \in V$ and $v$ extreme, so that $G^{*} H^{*}$ is the identity on a dense subset of $E \cup(-E)$. Consequently $G^{*}$ is, on $X$, a homeomorphism, and $H^{*}$ is, on $E \cup(-E)$, the inverse of this homeomorphism. From the structure of $E \cup(-E)$ it follows (see preliminary remarks) that $U^{c} \cap V^{c}$ and $\left[-\left(U^{c} \cup V^{c}\right)\right] \cap\left(U^{c} \cup V^{c}\right)$ are void, and it is also clear that $H^{*}$ maps $E$ homeomorphically onto $U^{c} \cup V^{c}$.

It remains to show that $H$ maps $B$ onto $C_{Y}$. The image $G^{*}(S)$ of the unit sphere $S$ of $B^{*}$ is convex and weak* compact, and each extreme point of the unit sphere $T$ of $C_{Y}^{*}$, as was shown in the preceding paragraph, belongs to $G^{*}(S)$. From the Krein-Milman theorem it follows that $T \subset G^{*}(S)$, and since $G^{*}$ has norm one, $T=G^{*}(S)$. Since $H^{*} G^{*}$ is the identity on $B^{*}$ and since $G^{*}$ maps $B^{*}$ onto $C_{Y}^{*}$, it follows that $H^{*}$ is $1-1$. Because $H^{*}$ is $1-1$ it is true that $H$ maps $B$ onto $C_{Y}$, for otherwise there is a nonzero linear functional on $C_{Y}$ which vanishes on the range of $H$ (a closed subspace) and $H^{*}$ applied to this functional gives the zero of $B^{*}$. The proof of the lemma is then complete. 
The theorem is now established as follows. Choose, using Zorn's Lemma, an open subset $W$ of $X$ maximal with respect to the property that $(-W)$ $\cap W$ be void. Then $(-W) \cup W$ is dense in $X$. Applying the lemma to $U=W$, $V=$ void set, it follows that $\left(-W^{c}\right) \cap\left(W^{c}\right)$ is void, and that $W^{c}$ is open as well as closed in $X$. Moreover $H$ is an isometry of $B$ onto $C_{Y}$, where $Y$ is homeomorphic to $W^{c}$. Proceeding, let $U$ be any open subset of $W^{c}$ and let $V=W^{c} \backslash U^{c}$. Applying the lemma again, we see that $U^{c} \cap V^{c}$ is void so that $U^{c}$ is open and it is proven that $W^{c}$ is extremally disconnected, which establishes the theorem.

\section{REFERENCES}

[AK] R. F. Arens and J. L. Kelley, Characterizations of the space of continuous functions over a compact Hausdorff space, Trans. Amer. Math. Soc. vol. 62 (1947) pp. 499-508.

[B] G. Birkhoff, Lattice theory, Amer. Math. Soc. Colloquium Publications, vol. 25, rev. ed., New York, 1948.

[G] D. B. Goodner, Projections in normed linear spaces, Trans. Amer. Math. Soc. vol. 69 (1950) pp. 89-108.

[HT] A. Horn and A. Tarski, Measures in Boolean algebras, Trans. Amer. Math. Soc. vol. 64 (1948) pp. 467-497.

[J] M. Jerison, Characterizations of certain spaces of continuous functions, Trans. Amer. Math. Soc. vol. 70 (1951) pp. 103-113.

[K] J. L. Kelley, Note on a theorem of Krein and Milman, Journ. of the Osaka Inst. Sci. and Tech. vol. 3 (1951) pp. 1-2.

[N] L. Nachbin, $A$ theorem of the Hahn-Banach type for linear transformations, Trans. Amer. Math. Soc. vol. 68 (1950) pp. 28-46.

TUlANE University,

New Orleans, La., 